\title{
Phase structure and real-time dynamics of the massive Thirring model in 1+1 dimensions using the tensor-network method*
}

\author{
Mari Carmen Bañuls ${ }^{a, b \dagger}$, Krzysztof Cichy ${ }^{c \ddagger}$, Hao-Ti Hung ${ }^{d \S}$, Ying-Jer Kao ${ }^{d \uparrow}$, \\ C.-J. David Lin $\| e, f * *$, Yu-Ping Lin ${ }^{g \dagger \dagger}$, David T.-L. Tan ${ }^{e \ddagger \ddagger}$
}

${ }^{a}$ Max Planck Institut für Quantenoptik, Garching 86748, Germany

${ }^{b}$ Munich Centre for Quantum Science and Technology, Munich 80799, Germany

${ }^{c}$ Faculty of Physics, Adam Mickiewicz University, ul. Uniwersytetu Poznańskiego 2, 61-614 Poznań, Poland

${ }^{d}$ Department of Physics, National Taiwan University, Taipei 10617 Taiwan

${ }^{e}$ Institute of Physics, National Chiao-Tung University, Hsinchu 30010, Taiwan

${ }^{f}$ Centre for High Energy Physics, Chung-Yuan Christian University, Chung-Li, 32032, Taiwan

${ }^{g}$ Department of Physics, University of Colorado, Boulder, CO 80309, USA

Email:banulsmempq.mpg.de, kcichy@amu.edu.pl, hunghaoti852@gmail.com,

yjkao@phys.ntu.edu.tw, dlin@mail.nctu.edu.tw,

Yuping. Lin@colorado.edu, tanlin2013.py04genctu.edu.tw

\begin{abstract}
We present concluding results from our study for zero-temperature phase structure of the massive Thirring model in 1+1 dimensions with staggered regularisation. Employing the method of matrix product states, several quantities, including two types of correlators, are investigated, leading to numerical evidence of a Berezinskii-Kosterlitz-Thouless phase transition. Exploratory results for real-time dynamics pertaining to this transition, obtained using the approaches of variational uniform matrix product state and time-dependent variational principle, are also discussed.
\end{abstract}

37th International Symposium on Lattice Field Theory - Lattice2019

16-22 June 2019

Wuhan, China

${ }^{*}$ The authors warmly thank Pochung Chen for discussions. Numerical simulations have been carried out on the LOEWE-CSC high-performance computer of Johann Wolfgang Goethe-University Frankfurt am Main, and on the highperformance computing facilities at National Chiao-Tung University.

$\dagger$ Paritally supported by the Deutsche Forschungsgemeinschaft (DFG, German Research Foundation) under Germany's Excellence Strategy - EXC-2111 - 390814868, and by the EU-QUANTERA project QTFLAG (BMBF grant No. 13N14780).

${ }^{\ddagger}$ Supported by National Science Centre (Poland) grant SONATA BIS 2016/22/E/ST2/00013.

${ }^{\S}$ Supported in part by Ministry of Science and Technology (MoST) of Taiwan under Grants No. 105-2112-M-002023-MY3.

I Supported in part by Ministry of Science and Technology (MoST) of Taiwan under Grants No. 105-2112-M-002023-MY3, 107-2112-M-002 -016-MY3, and 108-2918-I-002 -032.

"Speaker.

** Partially supported by Taiwanese MoST Grant No. 105-2628-M-009-003-MY4.

${ }^{\dagger \dagger}$ Sponsored by the Army Research Office under Grant No. W911NF-17-1-0482.

${ }^{\ddagger \ddagger}$ Supported by Taiwanese MoST Grant No. 105-2628-M-009-003-MY4. 


\section{Introduction}

Tensor-network (TN) methods, combined with lattice regularisation, have been applied to study quantum field theories (QFTs) in recent years [1]. These methods allow one to work directly with the Hamiltonian operator, without relying on Monte Carlo simulations for path integrals. Therefore, they offer opportunities for solving the sign problem, and for examining real-time dynamics.

This article presents the status of our research programme of implementing the TN strategy for the investigation of the massive Thirring model in $1+1$ dimensions. In particular, we resort to the formulation of matrix product states (MPS) in this work. The action of the field theory is

$$
S_{\mathrm{Th}}[\psi, \bar{\psi}]=\int d^{2} x\left[\bar{\psi} i \gamma^{\mu} \partial_{\mu} \psi-m \bar{\psi} \psi-\frac{g}{2}\left(\bar{\psi} \gamma_{\mu} \psi\right)\left(\bar{\psi} \gamma^{\mu} \psi\right)\right]
$$

with $m$ and $g$ being the fermion mass and the four-fermion coupling constant. In Refs. [2, 3], it was demonstrated that the sector of zero total fermion number in this model is dual to the sine-Gordon (SG) theory, which is also known to be connected to the two-dimensional classical XY model [4]. This means that a Berezinskii-Kosterlitz-Thouless (BKT) phase transition can exist in the Thirring model. According to perturbative analysis of the renormalisation-group (RG) flows [5], this transition occurs at a critical coupling $g_{*}$ with its value being $m$-dependent. It can be shown in perturbation theory that $g_{*}=\bar{g}_{*}=-\pi / 2$ at $m=0$, and $g_{*}$ decreases with increasing $m$. In the regime $g<g_{*}$, the theory in Eq. (1.1) is expected to be at criticality. Combining with the well-known fact that the (1+1)-dimensional massless Thirring model is a conformal field theory, one concludes that on the $g-m$ plane, there is a fixed line, $m=0$. This fixed line is divided into two parts, with $(g>-\pi / 2, m=0)$ being unstable and $(g<-\pi / 2, m=0)$ being stable.

In this contribution to the proceedings of the Lattice 2019 conference, we report concluding results from our study, using the MPS strategy, for equilibrium zero-temperature phase structure of the massive Thirring model in $1+1$ dimensions with staggered regularisation. These results have been published in Ref. [6]. Some aspects of this investigation were also presented at the Lattice 2017 and 2018 conferences [7, 8]. In addition, we describe our on-going efforts in examining real-time dynamics in the massive Thirring model in Sec. 4.

\section{Formulation and strategy}

The classical action in Eq. (1.1) is suitable for performing calculations employing the pathintegral formalism. However, in the MPS approach, one works with the Hamiltonian in the operator formulation of the theory. For this purpose, effects from the anomalous breaking of the vector symmetry in two dimensions have to be taken into account [9]. The Hamiltonian operator can be constructed through studying the commutation relations that are satisfied by the energy-momentum tensor [10], and then be discretised on a one-dimensional lattice using the staggered regularisation $[11,12]$. For convenience of carrying out numerical computations, in this work the fermionic degrees of freedom in this Hamiltonian are mapped onto spin operators through the Jordan-Wigner (JW) transformation. Details of the above procedure can be found in Ref. [6]. Implementation of this strategy turns the Hamiltonian operator of the continuum Thirring model, $H_{\mathrm{Th}}$, into that of the 
XXZ spin chain coupled to both uniform and staggered magnetic fields,

$$
\begin{aligned}
& H_{\mathrm{XXZ}}=\frac{v(g)}{a} \bar{H}_{\text {sim }}, \text { with } \bar{H}_{\mathrm{sim}}=-\frac{1}{2} \sum_{n=0}^{N-2}\left(S_{n}^{+} S_{n+1}^{-}+S_{n+1}^{+} S_{n}^{-}\right)+a \tilde{m}_{0} \sum_{n=0}^{N-1}(-1)^{n}\left(S_{n}^{z}+\frac{1}{2}\right) \\
& +\Delta(g) \sum_{n=0}^{N-1}\left(S_{n}^{z}+\frac{1}{2}\right)\left(S_{n+1}^{z}+\frac{1}{2}\right)
\end{aligned}
$$

where $a$ is the lattice spacing, $N$ is the total number of lattice sites, $S_{n}^{ \pm}=S_{n}^{x} \pm i S_{n}^{y}$ and $S_{n}^{z}$ are the spin matrices ( $S_{n}^{i}=\sigma^{i} / 2$ with $\sigma^{i}$ being the Pauli matrices) at the $n-$ th site, and $\left[S_{n}^{i}, S_{m}^{j}\right]_{n \neq m}=0$. The functions $v(g)$ and $\Delta(g)$ are the lattice version of wavefunction renormalisation and the fourfermion coupling [13],

$$
v(g)=\left(\frac{\pi-g}{\pi}\right) / \sin \left(\frac{\pi-g}{2}\right), \Delta(g)=\cos \left(\frac{\pi-g}{2}\right),
$$

and $\tilde{m}_{0}=m_{0} / v(g)$ with $m_{0}$ being the bare counterpart of the mass parameter, $m$, in Eq. (1.1). Since the $z$-component of the total spin corresponds to the total fermion number in the Thirring model, the Hamiltonian that we actually use in the simulations is

$$
\bar{H}_{\text {sim }}^{\text {penalty }}=\bar{H}_{\text {sim }}+\lambda\left(\sum_{n=0}^{N-1} S_{n}^{z}\right)^{2},
$$

which, upon choosing $\lambda$ to be large enough (100 in this work), ensures that the ground state obtained via a variational search is in the sector of vanishing total $S^{z}$ [14]. This enables us to interpret our results in terms of the dual SG theory and the XY model.

\section{Numerical results for the phase structure of the massive Thirring model}

To study the zero-temperature phase structure of the Thirring model, we scan the phase space by performing simulations with the Hamiltonian, $\bar{H}_{\text {sim }}^{\text {penalty }}$, in Eq. (2.3) at twenty-four values of the four-fermion coupling straddling the range $-0.9 \leq \Delta(g) \leq 1.0$, and at $a \tilde{m}_{0}=0,0.005,0.01,0.02$, 0.03, 0.04, 0.06, 0.08, 0.1, 0.13, 0.16, 0.2, 0.3, 0.4. Four system sizes, $N=400,600,800,1000$, are used. The search for the ground state is carried out with seven choices of the bond dimension, $D=50,100,200,300,400,500,600$. The target precision of this search for the ground-state energy is $10^{-7}$ in lattice units. The matrix product operator (MPO) for $\bar{H}_{\text {sim }}^{\text {penalty }}$, as well as details of our numerical implementation and analysis, can be found in Ref. [6].

As already reported at the Lattice 2018 conference [8], the von Neumann entanglement entropy, extracted by dividing the system of size $N$ into two subsystems between sites $n$ and $n+1$, is found to exhibit the conformal scaling behaviour [15] at $a \tilde{m}_{0}=0$. This scaling behaviour is also seen for $a \tilde{m}_{0} \neq 0$ when $g$ is smaller than a $a \tilde{m}_{0}$-dependent value, $g_{*}$.

To further probe the phase structure, in the past year we performed a detailed investigation of two types of correlation functions, namely the connected density-density, $\langle\bar{\psi}(x) \psi(x) \bar{\psi}(0) \psi(0)\rangle_{\mathrm{c}}$, and the fermion-antifermion, $\langle\bar{\psi}(x) \psi(0)\rangle$, correlators. These correlation functions can be written 

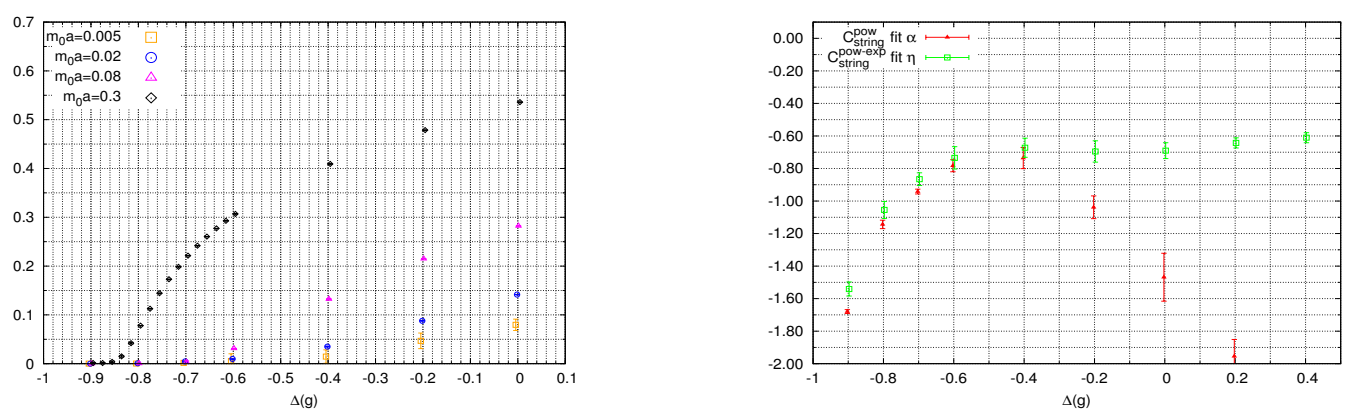

Figure 1: Results from fitting the fermion-antifermion correlator, $C_{\text {string }}(x)$, to the functions in Eq. (3.2). The system size is $N=1000$. Left: Values of $C$ at various choices of $a \tilde{m}_{0}$ with the power-exponential fit in Eq. (3.2). Right: Results of $\alpha$ and $\eta$ introduced in Eq. (3.2) at $a \tilde{m}_{0}=0.02$. Errors in the plots are from systematic effects as detailed in Ref. [6]

in terms of spin variables using the staggered-fermion discretisation and the JW transformation,

$$
\begin{aligned}
\langle\bar{\psi}(x) \psi(x) \bar{\psi}(0) \psi(0)\rangle_{\mathrm{c}} & \longrightarrow C_{z z}(x)=\frac{1}{N_{x}} \sum_{n}\left[\left\langle S_{n}^{z} S_{n+x}^{z}\right\rangle-\left\langle S_{n}^{z}\right\rangle\left\langle S_{n+x}^{z}\right\rangle\right], \\
\langle\bar{\psi}(x) \psi(0)\rangle & \longrightarrow C_{\text {string }}(x)=\frac{1}{N_{x}} \sum_{n}\left\langle S_{n}^{+} S_{n+1}^{z} \cdots S_{n+x-1}^{z} S_{n+x}^{-}\right\rangle,
\end{aligned}
$$

where the sum over $n$ means that at a given value of $x$, we average over all possible $C_{z z}(x)$ and $C_{\text {string }}(x)$ computed on the 200 -site subchain straddling the middle of the lattice. These two correlators are expected to decay with $x$ as a power law when the theory is at criticality, while the decay is (power-)exponentially cut-off in the gapped phase. We fit $C_{z z}(x)$ and $C_{\text {string }}(x)$ to the ansatzes

$$
\begin{aligned}
C^{\text {pow }}(x) & =\beta x^{\alpha}+C, \\
C^{\text {pow }-\exp }(x) & =B x^{\eta} A^{x}+C,
\end{aligned}
$$

as well as other multi-exponential functions. For both $C_{z z \text { string }}(x)$, our data show that for all values of $\Delta(g)$ at $a \tilde{m}_{0}=0$, as well as at $\Delta(g) \lesssim-0.7$ when $a \tilde{m}_{0} \neq 0$, the power-law and power-exponential fits are better compared to the multi-exponential fits. In this regime, it is observed that the parameter $A$ in Eq. (3.2) is consistent with unity, and the exponents $\alpha$ and $\eta$ are compatible with each other.

It is worth noting that the constant, $C$, in Eq. (3.2) is expected to be consistent with zero in $C_{z z}(x)$, and our data demonstrate this feature. For $C_{\text {string }}(x)$, this constant vanishes only in the critical phase. It is non-zero when the fermion mass is a relevant coupling in the field theory, corresponding to the appearance of the string order induced by the staggered magnetic field in the spin model $[16,17]$. This property is also seen in numerical results of this work. Figure 1 shows examples for fits of $C_{\text {string }}(x)$. From the plot on the left-hand side, it is obvious that we can identify a region on the $\Delta(g)-a \tilde{m}_{0}$ plane where $C=0$. This can be used to probe the phase structure. In the right-hand plot in Fig. 1, it is observed that the exponent, $\alpha$ (or $\eta$ ), depends on the four-fermion coupling, $g$, in the conformal phase, providing evidence that the phase transition is of the BKT-type. It is also noted that the power-law function does not result in good fits in the gapped phase.

Since the constant, $C$, in fitting $C_{\text {string }}(x)$ to the power-exponential function is the most accuratelydetermined parameter in our analysis, we use its value to probe the phase structure of the Thirring 

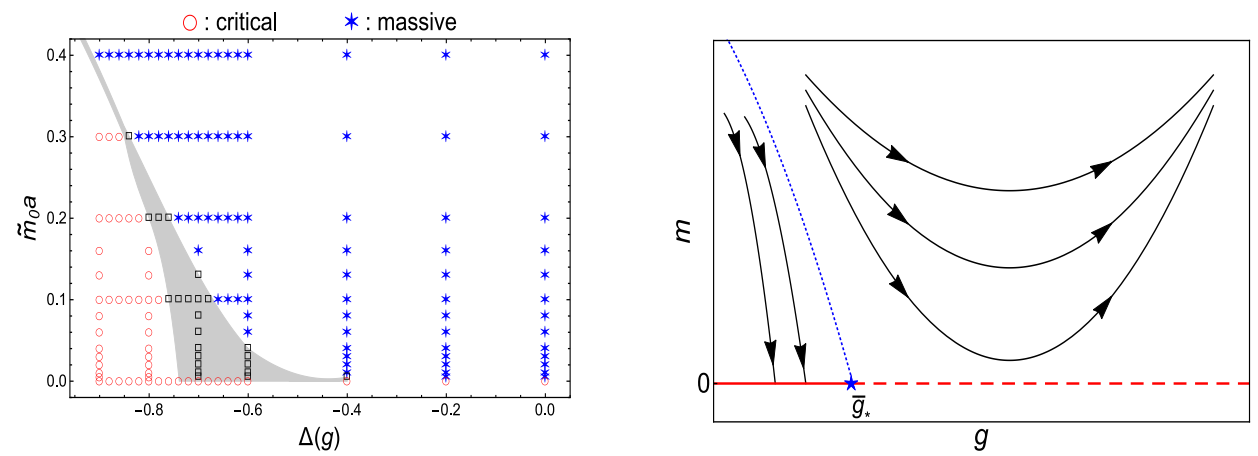

Figure 2: Left: Results of the phase structure of the massive Thirring model. Black boxes indicate points where our data are not precise enough to give clear answers. Right: Renormalisation group flows from perturbation theory.

model. Results of this analysis are shown in the left-hand plot in Fig. 2. In the regime where $C$ is consistent with zero, it is observed that the parameter $A$ is compatible with one. Also displayed in the same figure (right-hand plot) is the RG flow of the theory, obtained using perturbative expansion in $m$ and $\left(g-\bar{g}_{*}\right)$, with $\bar{g}_{*}=-\pi / 2$. We see that both our numerical simulation and perturbation theory predict the existence of a phase where the fermion mass is an irrelevant coupling.

Finally, we also compute the fermion bilinear condensate, $\chi=\langle\bar{\psi} \psi\rangle$, and observe that it can be non-zero when the theory is at criticality. This shows that $\chi$ cannot be an order parameter for the phase transition, giving more evidence that the transition is of the BKT-type.

\section{Real-time dynamics}

Our work on the phase structure of the model enables the investigation of real-time dynamics pertaining to "quenching" across the phase boundary. Regarding this aspect of the study, we exploit translational invariance in the thermodynamic limit. For one-dimensional systems, this allows for expressing an infinite-size quantum state as a uniform MPS (uMPS), which can be represented with one bulk tensor, $A_{l, r}^{i}$, that contains one physical index ( $i$ ) and two bond-dimension indices ( $l$ and $r$ ), together with appropriate boundary tensors when computing amplitudes and matrix elements [18, 19]. The ground state is then extracted using the variational algorithm introduced in Ref. [19]. For the real-time evolution of this infinite one-dimensional system, we resort to the method of time-dependent variational principle (TDVP) [20].

In probing the dynamical quantum phase transition (DQPT), we compute the return rate [21],

$$
G_{\text {return }}(t)=-\lim _{N \rightarrow \infty} \frac{1}{N} \ln \left(\left|\left\langle 0_{1}\left|\mathrm{e}^{-i H\left(a \tilde{m}_{0}^{(2)}, g^{(2)}\right) t}\right| 0_{1}\right\rangle\right|^{2}\right),
$$

where $H\left(a \tilde{m}_{0}^{(2)}, g^{(2)}\right)$ denotes the Hamiltonian, $\bar{H}_{\text {sim }}^{\text {penalty }}$, in Eq. (2.3) with the values of the couplings set to $a \tilde{m}_{0}=a \tilde{m}_{0}^{(2)}$ and $g=g^{(2)}$. The state, $\left|0_{1}\right\rangle$, is the vacuum of the Hamiltonian $H\left(a \tilde{m}_{0}^{(1)}, g^{(1)}\right)$. The return rate can be extracted by examining the spectrum of the "bulk transfer matrix",

$$
T_{i, j}(t)=i\{\stackrel{\sqrt{A(t)}}{A}\} j
$$



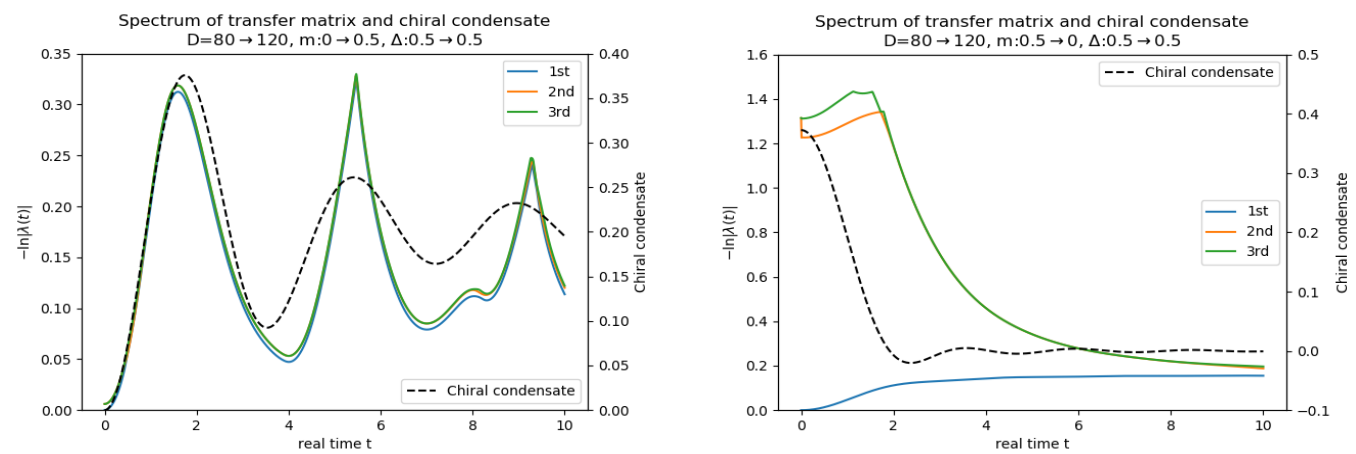

Figure 3: Real-time evolution of the largest three eigenvalues (generically denoted by $\lambda(t)$ ) of $T_{i, j}$. Left: Quenching from criticality to the gapped phase. Right: Quenching from the gapped phase to the conformal limit.

obtained by contracting the physical indices of the bulk tensors $\bar{A}_{0_{1}}$ and $A(t)$. The tensor $\bar{A}_{0_{1}}$ is the uMPS representation of $\left\langle 0_{1}\right|$, while $A(t)$ is that of $\exp \left[-i H\left(a \tilde{m}_{0}^{(2)}, g^{(2)}\right) t\right]\left|0_{1}\right\rangle$. Since the amplitude, $\left\langle 0_{1}\left|\exp \left[-i H\left(a \tilde{m}_{0}^{(2)}, g^{(2)}\right) t\right]\right| 0_{1}\right\rangle$, is obtained through infinite repetition of this transfer matrix, it is obvious that $G_{\text {return }}(t)$ can be determined using the largest eigenvalue of $T_{i, j}(t)$.

Dynamical quantum phase transitions are identified by non-analytic behaviour of $G_{\text {return }}(t)$ (or the largest eigenvalue of $T_{i, j}(t)$ as explained above) [21]. Figure 3 displays exploratory results of our study for such transitions in the massive Thirring model, using uMPS representation of ground states at bond dimension $D=80$. Numerical implementation is carried out with the Uni10 library [22]. We monitor the entanglement entropy, $S$, along the real-time evolution, and increase $D$ (with an upper bound $D=120$ ) when $S$ exhibits signs of being saturated. In these plots, the "chiral condensate" is computed by sandwiching the JW-transformed $\bar{\psi} \psi$ operator with real-time evolved states that are initially at $\left|0_{1}\right\rangle$. From the left-hand plot in Fig. 3, DQPTs are observed when $\left|0_{1}\right\rangle$ is the ground state of $H\left[a \tilde{m}_{0}^{(1)}, \Delta\left(g^{(1)}\right)\right]=H(0,0.5)$, and the real-time evolution is performed using $H\left[a \tilde{m}_{0}^{(2)}, \Delta\left(g^{(2)}\right)\right]=H(0.5,0.5)$. That is, DQPTs can occur while quenching from criticality into the gapped phase. Nevertheless, when quenching from the massive into the conformal limit, no DQPT is seen in this work hitherto (right-hand plot of Fig. 3). A similar scenario was also noted in condensed matter physics [23].

We examine further details of the observed DQPTs by extracting the largest five eigenvalues of $T_{i, j}$ around a transition point. Results of this study are displayed in Fig. 4. It is observed that this DQPT occurs when level-crossing between the largest and the second-largest eigenvalues happens. A viable physical picture of this phenomenon is currently being investigated.

\section{Conclusion and outlook}

This article reports final results of our MPS study for the zero-temperature phase structure of the massive Thirring model in $1+1$ dimensions. It is demonstrated that the approach is applicable for probing the BKT phase transition in the theory. We also show exploratory results for real-time dynamics in the model. Further numerical exploration for DQPTs, including those associated with the BKT phase transition that occurs at fixed, non-vanishing $a \tilde{m}_{0}$, is now being carried out. 

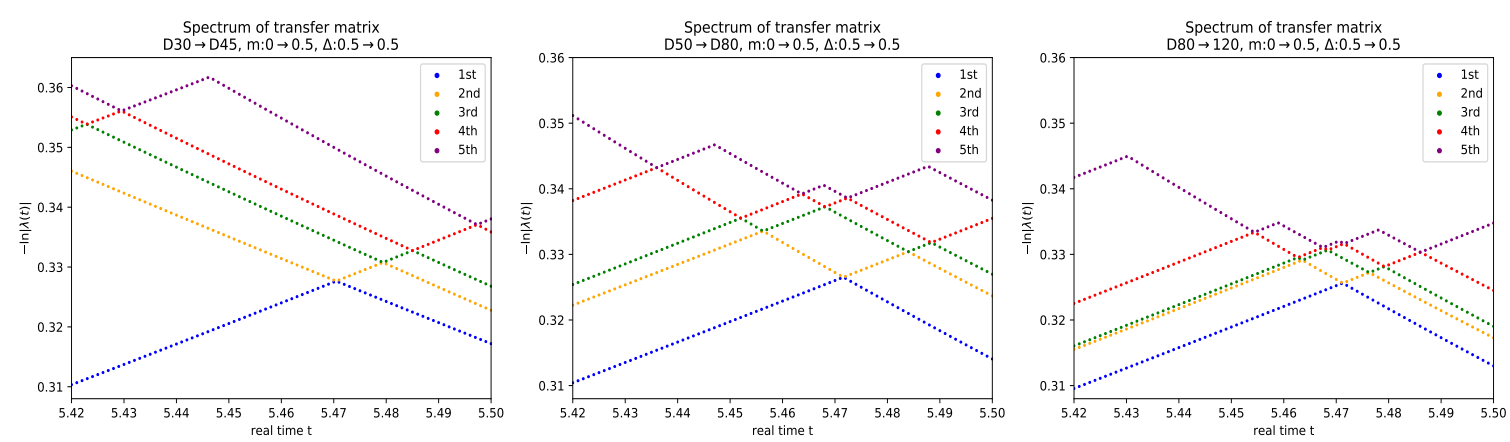

Figure 4: Details of real-time evolution of the largest 5 eigenvalues (generically denoted by $\lambda(t)$ ) of $T_{i, j}$, around a DQPT. The 3 plots correspond to different choices of bond dimension.

\section{References}

[1] M. C. Bañuls and K. Cichy, arXiv:1910.00257 [hep-lat].

[2] S. R. Coleman, Phys. Rev. D 11 (1975) 2088.

[3] S. Mandelstam, Phys. Rev. D 11 (1975) 3026.

[4] J. Jose, Phys. Rev. D 14 (1976) 2826.

[5] D. J. Amit, Y. Y. Goldschmidt and G. Grinstein, J. Phys. A 13 (1980) 585.

[6] M. C. Bañuls et al., Phys. Rev. D 100 (2019) 094504 [arXiv:1908.04536 [hep-lat]].

[7] M. C. Bañuls et al., EPJ Web Conf. 175 (2018) 11017 [arXiv:1710.09993 [hep-lat]].

[8] M. C. Bañuls et al., PoS LATTICE 2018 (2018) 229 [arXiv:1810.12038 [hep-lat]].

[9] J. S. Schwinger, Phys. Rev. 128 (1962) 2425.

[10] C. R. Hagen, Nuovo Cimento B51 (1967) 169.

[11] T. Banks, L. Susskind and J. B. Kogut, Phys. Rev. D 13 (1976) 1043.

[12] L. Susskind, Phys. Rev. D 16 (1977) 3031.

[13] A. Luther, Phys. Rev. B 14 (1976) 2153.

[14] M. C. Bañuls et al., JHEP 1311 (2013) 158 [arXiv:1305.3765 [hep-lat]].

[15] P. Calabrese and J. L. Cardy, J. Stat. Mech. 0406 (2004) P06002 [hep-th/0405152].

[16] D. Perez-Garcia et al., Phys. Rev. Lett. 100(16) (2008) 167202 [arXiv:0802.0447 [cond-mat.str-el]].

[17] M. Bortz et al., J. Phys. A 40 (2007) 4253 [arXiv:cond-mat/0612348].

[18] H. N. Phien et al., Phys. Rev. B 86 (2012) 245107 [arXiv:1207.0652 [quant-ph]].

[19] V. Zauner-Stauber et al., Phys. Rev. B 97 (2018) no.4, 045145 [arXiv:1701.07035 [quant-ph]].

[20] J. Haegeman et al., Phys. Rev. Lett. 107 (2011) 070601 [arXiv:1103.0936 [cond-mat.str-el]].

[21] M. Heyl et al., Phys. Rev. Lett. 110 (2013) 135704 [arXiv:1206.2505[cond-mat.stat-mech]].

[22] Y.-J. Kao, Y.-D. Hsieh and P. Chen, J. of Phys. Conf. Series 640 (2015) 012040.

[23] F. Andraschko and J. Sirker, Phys. Rev. B 89 (2014)125120 [arXiv:1312.4165 [cond-mat.str-el]]. 